J. Math. Kyoto Univ. (JMKYAZ)

$20-4$ (1980) 749-752

\title{
On the normality of $R(X)$
}

\author{
Dedicated to Professor Gorô Azumaya on his sixtieth birthday
}

By

Tomoharu AKIBA

(Received Sept. 10, 1979)

\section{Introduction.}

Throughout this paper, we understand by a ring a commutative ring with identity.

Let $R$ be a ring, and let $R[X]$ be the polynomial ring of an indeterminate $X$ over $R$. For an $f=f(X) \in R[X]$, we denote by $C(f)$, the ideal of $R$ generated by the coefficients of $f$. Let $N=N(R)=\{f \in R[X] \mid C(f)=R\}$. Then $N$ is a multiplicatively closed subset of $R[X]$, and we set $R(X)=R[X]_{N}$ (See [7]).

Let $T$ be a ring containing $R$, and let $S$ be the integral closure of $R$ in $T$. Let us consider the problem :

$(P)$ Is $S(X)$ the integral closure of $R(X)$ in $T(X)$ ? $^{1)}$

In [5], Gilmer and Hoffmann gave the affirmative answer to $(P)$ under an additional condition that $T[X]$ is quasi-normal. ${ }^{2}$. As be shown by an example in $\S 1$, the answer to $(P)$ is, in general, negative. We shall give a slight generalization of the result of Gilmer and Hoffmann. In $\S 2$, we shall consider the case where $T=Q(R)$ (=total quotient ring of $R$ ). Our main result in $\S 2$ is :

If $R$ is a quasi-normal noetherian ring, then $R(X)$ is integrally closed in $T(X)$.

The author expresses his hearty thanks to Professor M. Nagata for his valuable suggestions.

$\S 1$. We shall begin with an example.

Example. Let $k$ be a field, and let $U, V$ and $W$ be indeterminates. Set $R=k[U, V, W] /\left(U^{2}\right)=k[u, v, w]$, where $u, v$ and $w$ are the canonical images of $U, V$ and $W$ in $R$, respectively. Let $S$ be the integral closure of $R$ in $T=Q(R)$.

Now we shall show that $S(X)$ is not the integral closure of $R(X)$ in $T(X)$. Take $\alpha=u /(v X+w)$. Since $v$ and $w$ are non-zero-divisors in $R$ and, since $\alpha^{2}=0$, we see that $\alpha \in T(X)$ and integral over $R(X)$. So it is sufficient to show that

1) See Exercise 2 on page 415 in [4].

2) A ring is quasi-normal if it is integrally closed in its total quotient ring ([1], [2]). 
$\alpha \notin S(X)$. Suppose the contrary. Then there are an $f \in N(R)$ and $g^{*} \in S[X]$ such that $u /(v X+w)=g^{*} / f$ (see Lemma 1.2 below). From the fact that $u^{2}=0$, it follows that $g^{* 2}=0$ and, therefore $g^{*}$ is of the form $(u / d) g$, where $d$ is a non-zerodivisor of $R$ and $g \in R[X]$. Thus we get $u d f=u(v X+w) g$. Here we may take $d \in k[v, w]$ and $f, g \in k[v, w][X]$, as easily seen. Then we have that $d f=$ $(v X+w) g$, since every non-zero element of $k[v, w]$ is not a zero-divisor in $R$. On the other hand, the ring $k[v, w]$ is isomorphic to the polynomial ring $k[V, W]$, and, hence, $v X+w$ is a prime element of $k[v, w][X]$. Then it is clear that $C(f) \in(v, w)$, which contradicts the assumption that $f \in N(R)$.

The following lemma, which was suggested by Professor Nagata, is proved in a similar way as our proof of Theorem 3.2 in [1] and we omit the proof.

Lemma 1.1. Let $R$ be a ring, and let $\alpha$ be an element of $R(X)$ integral over $R[X]$. Then there exists an $h \in R[X]$ such that $\alpha-h$ is nilpotent in $R(X)$. In particular, if $R$ is reduced, $R[X]$ is integrally closed in $R(X)$.

The proof of the following lemma is found in [5].

Lemma 1.2. Let $R, S$ and $T$ be as in Introduction and let $N$ denote $N(R)$. Then: (i) $S[X]$ is the integral closure of $R[X]$ in $T[X]$, (ii) $S(X)\left(=S[X]_{N(S)}\right)$ $=S[X]_{N}$, and (iii) $S(X)$ is the integral closure of $R(X)$ in $T[X]_{N}$.

The following proposition is a slight generalization of the result of Gilmer and Hoffmann, because the reducedness of $T$ does not imply the quasi-normality of $T[X]$, in general, even if $T=Q(T)$. (See [1] and [3].)

Proposition 1.3. Let $R, S$ and $T$ be as in Introduction. If $T$ is reduced, then $S(X)$ is the integral closure of $R(X)$ in $T(X)$.

Proof. Let $\alpha$ be an element of $T(X)$ integral over $R(X)$. Then there is an $f \in N(R)$ such that $f \alpha$ is integral over $R[X]$, and hence, integral over $T[X]$. Since $f \alpha \in T(X), f \alpha$ is in $T[X]$ by virtue of Lemma 1.1. Then $f \alpha \in S[X]$ by Lemma 1.2 , whence $\alpha \in S[X]_{N(R)}=S(X)$.

$\S 2$. Throughout this section, we assume that $T=Q(R)$. Therefore, if $R$ is noetherian, $T(X)$ coincides with $Q(R[X])$.

Lemma 2.1. Assume that $R$ is a quasi-normal noetherian ring. Let $n$ be a non-zero nilpotent element of $R$, and let $M$ be a maximal ideal of $R$ which contains a non-zero-divisor. Then $M$ does not contain Ann $(n)$, where Ann $(n)=$ $\{r \in R \mid r n=0\}$.

Proof. Since $R_{M}$ is reduced by virture of Proposition 1.1 in [2], it is clear that $M \nsubseteq \operatorname{Ann}(n)$. 
Now we state our main result.

Theorem 2.2. If $R$ is a quasi-normal noetherian ring, then $R(X)$ is integrally closed in $T(X)$, that is, $R(X)$ is quasi-normal.

Proof. Let $\alpha$ be an element of $T(X)$ integral over $R(X)$. To show $\alpha \in R(X)$, we may assume that $\alpha$ is nilpotent by Lemma 1.1. Then, it is easy to see that we may restrict $\alpha$ to an element of the form $n / f$, where $n$ is a non-zero nilpotent element of $R$ and $f \in R[X]$ such that $C(f)$ contains a non-zero-divisor of $R$. Write $f=a_{0}+a_{1} X+\cdots+a_{n} X^{n}$ with $a_{i} \in R \quad(i=0,1, \cdots, n)$. Since $C(f)=$ $\left(a_{0}, a_{1}, \cdots, a_{n}\right),\left(a_{0}, a_{1}, \cdots, a_{n}\right)+\operatorname{Ann}(n)=R$ by virtue of Lemma 2.1. Take $b \in$ Ann $(n)$ so that $\left(a_{0}, a_{1}, \cdots, a_{n}, b\right)=R$. Then, setting $g=b+a_{0} X+\cdots+a_{n} X^{n+1}$, we get $n / f=n X / g$ with $C(g)=R$, which implies that $\alpha=n / f \in R(X)$. Thus the proof is complete.

Corollary 2.3. Let $R$ be a (not necessarily noetherian) ring, and let $S$ be the integral closure of $R$ in $T$. If $S$ is noetherian, then $S(X)$ is the integral closure of $R(X)$ in $T(X)$.

Proof. By Theorem 2.2, $S(X)$ is integrally closed in $T(X)$. On the other hand, since $S(X)$ is integral over $R(X)$ by virtue of Lemma 1.2, the corollary follows.

A ring is called a Prüfer ring if every finitely generated ideal containing a non-zero-divisor is invertible. A Prüfer ring is quasi-normal (see [6]).

Proposition 2.4. If $R$ is a Prüfer ring, then $R(X)$ is integrally closed in $T(X)$.

In order to prove the proposition, we need the following Lemma.

Lemma 2.5. Let $R$ be a ring, and let $f$ be an element of $R[X]$ such that $C(f)$ is invertible. Then for any $g \in R[X], C(f g)=C(f) C(g)$. (See Chap. IV in [4].)

Proof of Proposition 2.4. Take an $\alpha$ in $T(X)$ integral over $R(X)$. As in the proof of Theorem 2.2, we may assume that $\alpha$ is nilpotent and is of the form $n / f$, where $n$ is a nilpotent element of $R$ and $f \in R[X]$ such that $C(f)$ contains a non-zero-divisor of $R$. Write $f=a_{0}+a_{1} X+\cdots+a_{n} X^{n}$, and therefore, $C(f)=$ $\left(a_{0}, a_{1}, \cdots, a_{n}\right)$. Since $C(f)$ is invertible by our assumption, there are $b_{0} / s, b_{1} / s$, $\cdots, b_{n} / s$ in $C(f)^{-1}$ such that $\sum_{i} a_{i}\left(b_{i} / s\right)=1$, where $b_{i} \in R(i=0,1, \cdots, n)$ and $s$ is a non-zero-divisor of $R$. Let $g=b_{0}+b_{1} X+\cdots+b_{n} X^{n}$, and let $h=f g$. Then, by Lemma 2.5, $C(h)=C(f) C(g)=(s)$. Hence there is an $h^{\prime} \in R[X]$ such that $h=s h^{\prime}$ and $C\left(h^{\prime}\right)=R$, that is, $h^{\prime} \in N(R)$. Then $\alpha=n / f=n g /(f g)=(n / s) / h^{\prime} \in R(X)$, since $n / s$ is nilpotent and $R$ is quasi-normal. 
A Bezout ring is a ring such that every finitely generated ideal is principal. It is clear that a Bezout ring is a Prüfer ring. Hence we have:

Corollary 2.6. If $R$ is a Bezout ring, then $R(X)$ is integrally closed in $T(X)$.

\section{KYOTO UNIVERSITY}

\section{References}

[1] T. Akiba, Integrally-closedness of polynomial rings, Japan. J. Math., 6, 1980.

[2] T. Akiba and M. Nagata, On normality of a noetherian ring, J. Math. Kyoto Univ., 17, 1977.

[3] J.W. Brewer, D. L. Costa, and K. McCrimmon, Seminormality and root closure in polynomial rings and algebraic curves, J. Algebra, 58, 1979.

[4] R. Gilmer, Multiplicative Ideal Theory, Dekker, New York, 1972.

[5] R. Gilmer and J.F. Hoffmann, A characterization of Prufer domains in terms of polynomials, Pacific J. Math., 60, 1975.

[6] M.D. Larsen and P.J. McCarthy, Multiplicative Theory of Ideals, Academic Press, New York and London, 1971.

[7] M. Nagata, Local Rings, John Wiley, New York, 1962; reprint ed. Krieger, Huntingon, 1974. 